DE GRUYTER DOI 10.2478/v10129-011-0073-3

${ }^{1}$ O.A. Oduwaye, ${ }^{1}$ D.K. Ojo, ${ }^{2}$ J. Mkumbira, ${ }^{1}$ C.O. Alake, ${ }^{3}$ O. Adenuga, ${ }^{3}$ E.F.Mapayi

${ }^{1}$ Department of Plant Breeding and Seed Technology, Federal University of Agriculture (FUNAAB), PMB 2240, Abeokuta, Nigeria; ${ }^{2}$ International Institute of Tropical Agriculture (IITA), PMB 5320, Ibadan, Nigeria; ${ }^{3}$ Plant Breeding Section, Cocoa Research Institute of Nigeria, P.M.B. 5244 Ibaden, Nigeriae-mail oduwayeoa@unaab.edu.ng

\title{
GENETIC ASSESSMENT OF 23 CASSAVA, MANIHOT ESCULENTA CRANTZ, GENOTYPES AT TWO AGRO-CLIMATIC ZONES IN NIGERIA
}

\begin{abstract}
This study investigated genetic diversity among 23 cassava genotypes in two-agro-ecological zones (Ibadan and Mokwa), Nigeria. The experiment was conducted using a randomized complete block design with three replicates. The cassava genotypes were evaluated for tuber yield, fresh weight of tuber, number of tubers, tuber girth and length, dry matter and chlorophyll concentration. The data collected were subjected to analysis of variance and differences among the genotypes were computed using Duncan's multiple range test. Single linkage cluster and FASTCLUS analysis were used to group the cassava genotypes. Genotypic and phenotypic coefficient of variability, heritability and genetic advance were also evaluated. Genotype, environment and genotype $\times$ environment interaction $(\mathrm{GEI})$ were significant $(\mathrm{P}<0.01)$ for most of the traits evaluated. The magnitude of the environment was higher compared to genotype and GEI. Comparative mean performance varied from location to location. Tuber yield ranged from 0.32 for $92 / 0325$ to $0.90 \mathrm{~kg}$ for $99 / 3073$ with mean of $0.58 \mathrm{~kg}$, and $0.16 \mathrm{~kg}$ for $82 / 00058$ to $0.67 \mathrm{~kg}$ for $98 / 0581$ with mean of $0.35 \mathrm{~kg}$ in Mokwa. Higher genotypic and phenotypic coefficients of variability were observed in Ibadan than Mokwa, for most of the characters. The interrelationships among the characters revealed the superiority of some cassava genotypes for a character in one location and not in the other location. However, breeding potentials exists among the cassava genotypes across the two environments.
\end{abstract}

Key word: cassava, FASTCLUS, morphological performance, $\mathrm{G} \times$ E interaction

\section{INTRODUCTION}

Cassava (Manihot esculenta Crantz) is a perennial shrub widely grown in tropical Africa, Asia and Latin America (Olsen and Schaal, 2001). Nigeria

Communicated by Andrzej Aniot 
is the highest cassava producer in the world, producing a third more than Brazil and almost double the production capacity of Thailand and Indonesia. She produces about 38 million metric tonnes (MT) per annum, a figure expected to double by 2020 (FAO, 2002).

Cassava is the developing world's fourth most important crop, with production in 2006 estimated at 226 million tones (FAO, 2006). It produces bulky storage roots with a heavy concentration of carbohydrates. Apart from food, cassava derivatives and starch are used as confectionery, sweeteners, glues, plywood, textiles, paper, biodegradable products, monosodium glutamate and drugs.

In Nigeria, cassava is one of the most important staple foods, and the cheapest main source of dietary food energy for nearly one-third of the population of sub-Saharan Africa (Nweke et al., 2002; Tsegia et al., 2002). Currently, the government of Nigeria considers a transition from the present status of usage to the level of industrial raw material and livestock feed as a development goal that can spur growth with increase in employment. This consideration, therefore, emphasized the need for various research geared towards cassava improvement and production. The objectives of this study are to assess the influence of environment and genotypic differences on root yield and related traits.

\section{MATERIALS AND METHODS}

The experiment was conducted at two locations in Nigeria, Ibadan (Derived savanna) and Mokwa (Southern guinea savanna) Table 1. Twentythree cassava genotypes from International Institute of Tropical Agriculture (IITA), Nigeria were used for the study. The experimental design was a randomized complete block design with three replicates at each site under rain-fed conditions. Each plot consisted of 60 plants in ten-plant rows. The ridges were $1 \mathrm{~m}$ apart, $30 \mathrm{~cm}$ high and $6 \mathrm{~m}$ long. Spacing between plants was $1 \mathrm{~m}$. Weeding was done as necessary. At harvest $(12$ months after planting), data were collected from the inner plants within a plot for tuber length and diameter, fresh tuber yield, number of tubers and dry matter weight. Dry matter weights of tuberous roots were determined from a random bulk sample of five plants selected from the inner rows. The roots were peeled and shredded after washing. One hundred grams of fresh root (cortex) was taken in the form of chips and dried at $60^{\circ} \mathrm{C}$ for $48 \mathrm{~h}$ in a forced air oven. The dried samples were then reweighed to obtain the dry weights. Dry tuber yield was estimated by multiplying fresh storage tuber yield by dry matter content (Kawano et al., 1987). Chlorophyll concentration was measured using a Minolta chlorophyll meter. 
Description of the agro-ecologies of the trial locations

\begin{tabular}{lcc}
\hline \multicolumn{1}{c}{ Geographical data } & Ibadan & Mokwa \\
\hline Ecological zone & Forest savannah transition & Sub-humid Southern Guinea savannah \\
Longitude & $3^{\circ} 01^{\prime} \mathrm{W}$ & $11^{\circ} 30^{\prime} \mathrm{E}$ \\
Latitude & $7^{\circ} 29^{\prime} \mathrm{N}$ & $7^{\circ} 22^{\prime} \mathrm{S}$ \\
Altitude [m.a.s. 1$]$ & 215 & 161 \\
Minimum temperature $\left[{ }^{\circ} \mathrm{C}\right]$ & 12 & 13 \\
Maximum temperature $\left[{ }^{\circ} \mathrm{C}\right]$ & 34 & 36 \\
Mean annual rainfall $[\mathrm{mm}]$ & 1300 & 1150 \\
Soil type & Ferric-luvisols & Ferric-luvisols \\
\hline
\end{tabular}

Analysis of variance was performed using the GLM procedure of Statistical Analysis System (SAS, 9.2) and differences among the genotypes were computed using Duncan's multiple range test. The genetic and phenotypic variance components were used to compute the genotypic and phenotypic coefficients of variability, heritability (in the broad-sense) and genetic advance (Kumar et al., 1985; Allard, 1960)

$$
\text { Genotypic coefficient of variability }=\frac{\delta_{g}}{\bar{X}} \times 100
$$

Phenotypic coefficient of variability $=\frac{\delta_{p h}}{\bar{X}} \times 100$

$$
\begin{gathered}
H_{B}=\frac{\delta_{g}^{2}}{\delta_{g}^{2}+\delta_{e}^{2}} \\
\text { Genetic advance }=H_{B} \times K \times \delta_{p h}
\end{gathered}
$$

where

$\delta_{g}$ - genotypic standard deviation

$\delta_{p h}$ - phenotypic standard deviation

$H_{B}$ - heritability

$\bar{X}$ - grand mean for the character under consideration. 
$\delta^{2}{ }_{g}-$ genotypic variance

$\delta_{e}^{2}$ - environmental variance.

$K$ is selection differential $=2.06$ (assuming 5\% selection)

\section{RESULTS}

The combined analysis of variance across two environments (Table 2) showed significant $(\mathrm{P} \leq 0.01)$ genotype and location effects for chlorophyll concentration, tuber diameter and length, number of tubers, dry matter and dry tuber yield. Genotype $\times$ environment interaction (GEI) showed significant mean squares $(\mathrm{P} \leq 0.01)$ for all traits evaluated except number of tubers and fresh weight of tubers. Also, the table revealed that the relative magnitude of the environmental effect (as a proportion of the total sum of squares) was higher compared to genotype and GEI effects.

Table 2

Mean squares from combined analysis of variance of yield and related characters in 23 cassava genotypes across two locations

\begin{tabular}{lcccccccc}
\hline \multicolumn{1}{c}{ Source } & DF & $\begin{array}{c}\text { Chlorophyll } \\
\text { concentration }\end{array}$ & $\begin{array}{c}\text { Tuber } \\
\text { diameter } \\
{[\mathrm{cm}]}\end{array}$ & $\begin{array}{c}\text { Tuber } \\
\text { length }[\mathrm{cm}]\end{array}$ & $\begin{array}{c}\text { Number of } \\
\text { tubers/plant }\end{array}$ & $\begin{array}{c}\text { Fresh weight of } \\
\text { tubers per plant } \\
{[\mathrm{kg}]}\end{array}$ & $\begin{array}{c}\text { Dry matter } \\
{[\mathrm{kg}]}\end{array}$ & $\begin{array}{c}\text { Tuber yield } \\
\text { per plant } \\
{[\mathrm{kg}]}\end{array}$ \\
\hline Block & 4 & 6.56 & $3.42^{* *}$ & $950.81^{* *}$ & $13.97^{* *}$ & $2.14^{* *}$ & $0.0003^{* *}$ & $0.15^{* *}$ \\
Genotype(G) & 22 & $28.70^{* *}$ & $1.25^{* *}$ & $229.77^{* *}$ & $5.27^{* *}$ & $1.49^{* *}$ & $0.0002^{* *}$ & $0.07^{* *}$ \\
Environment(E) & 1 & $290.73^{* *}$ & $144.54^{* *}$ & $497.42^{* *}$ & $230.83^{* *}$ & 2.08 & $0.0085^{* *}$ & $1.90^{* *}$ \\
$\mathrm{G} \times \mathrm{E}$ & 22 & $18.61^{* *}$ & $1.34^{* *}$ & $192.78^{* *}$ & 3.09 & 0.81 & $0.0002^{* *}$ & $0.06^{* *}$ \\
Error & 88 & 5.83 & 0.44 & 42.83 & 2.26 & 0.62 & 0.00003 & 0.02 \\
$\mathrm{CV}(\%)$ & & 6.82 & 12.46 & 23.88 & 25.97 & 33.89 & 13.96 & 32.27 \\
\hline
\end{tabular}

Block $=$ block within location; $* *$ significant at $1 \%$ probability level

Tables 3 and 4 showed the mean performance for the 23 cassava genotypes at Ibadan and Mokwa, respectively. Tuber yield ranged from 0.32 for $92 / 0325$ to $0.90 \mathrm{~kg}$ for $99 / 3073$ with mean of $0.58 \mathrm{~kg}$, and $0.16 \mathrm{~kg}$ for $82 / 00058$ to $0.67 \mathrm{~kg}$ for $98 / 0581$ with mean of $0.35 \mathrm{~kg}$ in Mokwa. Thirteen (13) of the genotypes had above average yield in Ibadan and eleven (11) in Mokwa. Genotypes 96/0289 and TME419 had the highest tuber weight of $3.70 \mathrm{~kg}$ and dry matter of $0.067 \mathrm{~kg}$, respectively, at Ibadan. However, genotypes 98/0581 and 96/1089A had the highest tuber weight $(4.40 \mathrm{~kg})$ and dry matter $(0.050 \mathrm{~kg})$, respectively at Mokwa. The average number of tubers varied significantly among the genotypes at Mokwa and ranged from 4 (98/0510) to $9(97 / 4769)$ with average number of 7 tubers. However, it varies from $3(90 / 0325)$ to $7(96 / 0289)$ with average number of 5 tubers per plant in Ibadan. 
Table 3

Mean performance of the 23 cassava genotypes evaluated for root yields and its component at Ibadan

\begin{tabular}{|c|c|c|c|c|c|c|c|}
\hline Variety & $\begin{array}{l}\text { Chlorophyll } \\
\text { concentration }\end{array}$ & $\begin{array}{l}\text { Tuber diame- } \\
\text { ter }[\mathrm{cm}]\end{array}$ & $\begin{array}{l}\text { Tuber length } \\
\text { [cm] }\end{array}$ & $\begin{array}{c}\text { Number of } \\
\text { tubers per } \\
\text { plant }\end{array}$ & $\begin{array}{c}\text { Fresh weight } \\
\text { of tubers/plant } \\
{[\mathrm{kg}]}\end{array}$ & $\begin{array}{c}\text { Dry matter } \\
{[\mathrm{kg}]}\end{array}$ & $\begin{array}{c}\text { Tuber yield/ } \\
\text { plant }[\mathrm{kg}]\end{array}$ \\
\hline $99 / 3073$ & $35.57^{\mathrm{ad}}$ & $6.30^{\mathrm{de}}$ & $29.00^{\mathrm{fg}}$ & $6.34^{\mathrm{ac}}$ & $3.24^{\mathrm{ab}}$ & $0.056^{b-d}$ & $0.90^{\mathrm{a}}$ \\
\hline TME419 & $34.47^{b-d}$ & $6.35^{\mathrm{de}}$ & $33.33^{\text {ef }}$ & $4.40^{b-d}$ & $2.46^{\mathrm{a}-\mathrm{f}}$ & $0.067^{\mathrm{a}}$ & $0.82^{\mathrm{ab}}$ \\
\hline $95 / 0289$ & $38.63^{\mathrm{a}}$ & $8.20^{\mathrm{a}}$ & $56.00^{\mathrm{ab}}$ & $7.22^{\mathrm{a}}$ & $3.70^{\mathrm{a}}$ & $0.044^{\mathrm{h}-\mathrm{j}}$ & $0.81^{\mathrm{ab}}$ \\
\hline $82 / 00058$ & $33.93^{b-d}$ & $8.60^{\mathrm{a}}$ & $32.00^{\mathrm{f}}$ & $6.82^{\mathrm{ab}}$ & $3.22^{\mathrm{ab}}$ & $0.042^{\mathrm{h}-\mathrm{j}}$ & $0.68^{\mathrm{acc}}$ \\
\hline $96 / 1565$ & $34.00^{b-d}$ & $7.60^{\mathrm{ac}}$ & $40.67^{\text {de }}$ & $4.11^{b-d}$ & $3.02^{\mathrm{a-d}}$ & $0.044^{\mathrm{h}-\mathrm{j}}$ & $0.67^{\mathrm{acc}}$ \\
\hline M98/0040 & $39.40^{\mathrm{a}}$ & $6.35^{\mathrm{de}}$ & $22.33^{\text {gh }}$ & $4.70^{\mathrm{add}}$ & $2.62^{\mathrm{ae}}$ & $0.050^{\mathrm{c}-\mathrm{g}}$ & $0.65^{\mathrm{ac}}$ \\
\hline 30572 & $28.37^{\mathrm{ef}}$ & $6.20^{\mathrm{de}}$ & $25.67^{\mathrm{fg}}$ & $5.88^{\mathrm{acc}}$ & $3.15^{\mathrm{ac}}$ & $0.041^{\mathrm{ij}}$ & $0.64^{\mathrm{acc}}$ \\
\hline $98 / 0581$ & $33.38^{b-d}$ & $7.65^{\mathrm{ac}}$ & $52.67^{\mathrm{acc}}$ & $5.41^{\mathrm{ad}}$ & $2.86^{\mathrm{ad}}$ & $0.045^{\mathrm{g} j}$ & $0.64^{\mathrm{acc}}$ \\
\hline $96 / 1632$ & $36.40^{\mathrm{a}-\mathrm{c}}$ & $6.67^{\mathrm{ce}}$ & $41.33^{\mathrm{de}}$ & $4.23^{\mathrm{b}-\mathrm{d}}$ & $2.03^{\mathrm{b-f}}$ & $0.063^{\mathrm{ab}}$ & $0.64^{\mathrm{acc}}$ \\
\hline $97 / 4769$ & $26.27^{\mathrm{f}}$ & $6.43^{\mathrm{de}}$ & $28.33^{\text {fg }}$ & $3.95^{\mathrm{cd}}$ & $2.20^{\mathrm{b-f}}$ & $0.058^{\mathrm{bc}}$ & $0.64^{\mathrm{acc}}$ \\
\hline $98 / 0510$ & $38.73^{\mathrm{a}}$ & $8.00^{\mathrm{ab}}$ & $45.67^{\mathrm{cd}}$ & $4.09^{b-d}$ & $2.66^{\mathrm{ae}}$ & $0.046^{\mathrm{e}-\mathrm{j}}$ & $0.63^{\mathrm{ac}}$ \\
\hline $4(2) 1425$ & $31.70^{\mathrm{de}}$ & $7.00^{\text {be }}$ & $57.67^{\mathrm{a}}$ & $3.79^{\mathrm{cd}}$ & $2.26^{\mathrm{b-f}}$ & $0.053^{\mathrm{c}-\mathrm{g}}$ & $0.60^{b-d}$ \\
\hline $92 \mathrm{~B} / 00068$ & $34.53^{\mathrm{b}-\mathrm{d}}$ & $7.00^{\text {be }}$ & $15.33^{\mathrm{h}}$ & $3.67^{\mathrm{cd}}$ & $2.48^{\mathrm{a}-\mathrm{f}}$ & $0.048^{\mathrm{e}-\mathrm{j}}$ & $0.60^{b-d}$ \\
\hline $91 / 02324$ & $33.17^{b-d}$ & $6.00^{\mathrm{ef}}$ & $45.00^{\mathrm{cd}}$ & $5.29^{\mathrm{add}}$ & $2.71^{\mathrm{ae}}$ & $0.042^{\mathrm{h}-\mathrm{j}}$ & $0.56^{b-d}$ \\
\hline $96 / 1089 A$ & $37.00^{\mathrm{ab}}$ & $6.33^{\mathrm{de}}$ & $49.00^{b-d}$ & $5.64^{\mathrm{acc}}$ & $2.76^{\mathrm{ae}}$ & $0.041^{\mathrm{h}-\mathrm{j}}$ & $0.56^{\mathrm{bc}}$ \\
\hline $98 / 0505$ & $28.53^{\text {ef }}$ & $6.43^{\mathrm{de}}$ & $31.67^{\mathrm{f}}$ & $3.64^{\mathrm{cd}}$ & $2.00^{b-f}$ & $0.045^{\mathrm{f}-\mathrm{j}}$ & $0.53^{b-c}$ \\
\hline $97 / 0162$ & $33.10^{b-d}$ & $7.00^{\text {be }}$ & $30.67^{\mathrm{fg}}$ & $3.91^{\mathrm{cd}}$ & $2.11^{b-f}$ & $0.045^{\mathrm{f}-\mathrm{j}}$ & $0.47^{\mathrm{c-g}}$ \\
\hline $94 / 0034$ & $33.97^{b-d}$ & $6.23^{\mathrm{de}}$ & $29.00^{\mathrm{fg}}$ & $4.93^{\mathrm{ad}}$ & $2.23^{b-f}$ & $0.038^{j}$ & $0.43^{\mathrm{cd}}$ \\
\hline $96 / 0603$ & $32.38^{\mathrm{ce}}$ & $6.43^{\mathrm{de}}$ & $29.00^{\mathrm{fg}}$ & $5.44^{\mathrm{add}}$ & $1.93^{\mathrm{c}-\mathrm{f}}$ & $0.043^{\mathrm{h}-\mathrm{j}}$ & $0.42^{\mathrm{cd}}$ \\
\hline $96 / 1642$ & $31.63^{\mathrm{de}}$ & $6.40^{\mathrm{de}}$ & $33.00^{\mathrm{ef}}$ & $5.36^{\mathrm{add}}$ & $1.54^{\mathrm{ef}}$ & $0.054^{\text {ce }}$ & $0.41^{\mathrm{cd}}$ \\
\hline $92 / 0067$ & $31.43^{\text {de }}$ & $7.10^{\text {be }}$ & $30.00^{\mathrm{fg}}$ & $4.02^{\mathrm{cd}}$ & $1.81^{\mathrm{d}-\mathrm{f}}$ & $0.043^{\mathrm{h}-\mathrm{j}}$ & $0.39^{\mathrm{cd}}$ \\
\hline M98/0028 & $34.10^{b-d}$ & $5.07^{\mathrm{f}}$ & $28.67^{\mathrm{fg}}$ & $4.37^{\mathrm{b}-\mathrm{d}}$ & $1.83^{\mathrm{d}-\mathrm{f}}$ & $0.042^{\mathrm{h}-\mathrm{j}}$ & $0.38^{\mathrm{cd}}$ \\
\hline $92 / 0325$ & $31.70^{\mathrm{de}}$ & $6.40^{\mathrm{de}}$ & $26.33^{\text {fg }}$ & $2.73^{\mathrm{d}}$ & $1.31^{\mathrm{f}}$ & $0.049^{\mathrm{d}-\mathrm{h}}$ & $0.32^{\mathrm{d}}$ \\
\hline Mean & $33.58 \pm 0.45$ & $6.76 \pm 0.11$ & $35.32 \pm 1.39$ & $4.78 \pm 0.19$ & $2.44 \pm 0.10$ & $0.05 \pm 0.001$ & $0.58 \pm 0.02$ \\
\hline
\end{tabular}

Means followed by the same letter within the column are not significantly different $(\mathrm{P} \leq 0.05)$ using DMRT

The highest concentration of chlorophyll was recorded for genotypes M98/0040 (39.40), 98/0510 (38.73) and 95/0289 (38.63) at Ibadan while $99 / 3073$ had the highest chlorophyll concentration of 41.70 at Mokwa. 
Table 4

Mean performance of the 23 cassava genotypes evaluated for root yields and its component at Mokwa

\begin{tabular}{|c|c|c|c|c|c|c|c|}
\hline Variety & $\begin{array}{l}\text { Chlorophyll } \\
\text { concentration }\end{array}$ & $\begin{array}{c}\text { Tuber diameter } \\
{[\mathrm{cm}]}\end{array}$ & $\begin{array}{l}\text { Tuber length } \\
{[\mathrm{cm}]}\end{array}$ & $\begin{array}{l}\text { Number of } \\
\text { tubers per plant }\end{array}$ & $\begin{array}{c}\text { Fresh weight o } \\
\text { tubers/plant } \\
{[\mathrm{kg}]}\end{array}$ & f & $\begin{array}{c}\text { Tuber yield/ } \\
\text { plant }[\mathrm{kg}]\end{array}$ \\
\hline 99/3073 & $41.70^{\mathrm{a}}$ & $4.77^{\mathrm{ac}}$ & $35.67^{\mathrm{a}}$ & $7.14^{\text {add }}$ & $2.10^{\mathrm{bc}}$ & $0.036^{\mathrm{cd}}$ & $0.38^{\text {be }}$ \\
\hline TME 419 & $37.37^{\mathrm{ab}}$ & $4.57^{\mathrm{ac}}$ & $32.67^{\mathrm{a}}$ & $8.84^{\mathrm{ab}}$ & $2.66^{\mathrm{b}}$ & $0.018^{\mathrm{h}}$ & $0.23^{\mathrm{de}}$ \\
\hline $95 / 0289$ & $37.63^{\mathrm{ab}}$ & $4.37^{\mathrm{bc}}$ & $32.00^{\mathrm{a}}$ & $7.00^{\text {add }}$ & $2.60^{\mathrm{bc}}$ & $0.029^{\mathrm{gf}}$ & $0.36^{\mathrm{ce}}$ \\
\hline $82 / 00058$ & $31.93^{\mathrm{c}}$ & $3.97^{\mathrm{bc}}$ & $26.00^{\mathrm{a}}$ & $7.57^{\mathrm{ac}}$ & $1.94^{\mathrm{bc}}$ & $0.017^{\mathrm{h}}$ & $0.16^{\mathrm{e}}$ \\
\hline $96 / 1565$ & $38.00^{\mathrm{ab}}$ & $4.90^{\mathrm{ac}}$ & $41.00^{\mathrm{a}}$ & $7.00^{\mathrm{ac}}$ & $2.23^{\mathrm{bc}}$ & $0.033^{\mathrm{d} f \mathrm{f}}$ & $0.38^{\text {be }}$ \\
\hline M98/0040 & $38.67^{\mathrm{ab}}$ & $4.77^{\mathrm{ac}}$ & $29.67^{\mathrm{a}}$ & $9.17^{\mathrm{ab}}$ & $2.35^{\mathrm{bc}}$ & $0.026^{\text {gf }}$ & $0.29^{\text {be }}$ \\
\hline 30572 & $35.50^{\mathrm{bc}}$ & $5.00^{\mathrm{ac}}$ & $39.00^{\mathrm{a}}$ & $8.48^{\mathrm{ac}}$ & $2.07^{\mathrm{bc}}$ & $0.031^{\mathrm{d}-\mathrm{f}}$ & $0.32^{\text {ce }}$ \\
\hline 98/0581 & $37.63^{\mathrm{ab}}$ & $4.53^{\mathrm{ac}}$ & $29.33^{\mathrm{a}}$ & $7.00^{\mathrm{abc}}$ & $4.40^{\mathrm{a}}$ & $0.032^{\mathrm{d}-\mathrm{f}}$ & $0.67^{\mathrm{a}}$ \\
\hline $96 / 1632$ & $35.30^{\mathrm{bc}}$ & $4.67^{\mathrm{ac}}$ & $31.33^{\mathrm{a}}$ & $7.67^{\mathrm{ac}}$ & $1.86^{\mathrm{bc}}$ & $0.043^{\mathrm{b}}$ & $0.39^{\mathrm{ae}}$ \\
\hline $97 / 4769$ & $39.07^{\mathrm{ab}}$ & $5.20^{\mathrm{ac}}$ & $31.33^{\mathrm{a}}$ & $9.27^{\mathrm{a}}$ & $2.89^{\mathrm{bc}}$ & $0.044^{\mathrm{ab}}$ & $0.66^{\mathrm{ab}}$ \\
\hline $98 / 0510$ & $38.57^{\mathrm{ab}}$ & $5.30^{\mathrm{ab}}$ & $26.33^{\mathrm{a}}$ & $4.17^{\mathrm{d}}$ & $0.97^{\mathrm{c}}$ & $0.032^{\mathrm{d}-\mathrm{f}}$ & $0.15^{\mathrm{e}}$ \\
\hline $4(2) 1425$ & $35.13^{\mathrm{bc}}$ & $4.53^{\mathrm{ac}}$ & $31.33^{\mathrm{a}}$ & $6.00^{b-d}$ & $2.06^{\mathrm{bc}}$ & $0.034^{\mathrm{de}}$ & $0.36^{\mathrm{ce}}$ \\
\hline $92 \mathrm{~B} / 00068$ & $37.27^{\mathrm{ab}}$ & $4.97^{\mathrm{ac}}$ & $28.67^{\mathrm{a}}$ & $6.50^{\text {ad }}$ & $1.76^{\mathrm{bc}}$ & $0.027^{\mathrm{e}-\mathrm{g}}$ & $0.23^{\mathrm{de}}$ \\
\hline $91 / 02324$ & $35.37^{\mathrm{bc}}$ & $5.27^{\mathrm{ab}}$ & $32.33^{\mathrm{a}}$ & $8.00^{\mathrm{ac}}$ & $2.52^{\mathrm{bc}}$ & $0.033^{\mathrm{df} f}$ & $0.41^{\text {ae }}$ \\
\hline $96 / 1089 \mathrm{~A}$ & $37.76^{\mathrm{ab}}$ & $4.33^{\mathrm{bc}}$ & $38.33^{\mathrm{a}}$ & $7.20^{\text {add }}$ & $2.22^{\mathrm{bc}}$ & $0.050^{\mathrm{a}}$ & $0.44^{\mathrm{ae}}$ \\
\hline $98 / 0505$ & $36.47^{\mathrm{bc}}$ & $5.77^{\mathrm{a}}$ & $35.00^{\mathrm{a}}$ & $7.25^{\text {ad }}$ & $2.15^{\mathrm{bc}}$ & $0.044^{\mathrm{ab}}$ & $0.47^{\text {add }}$ \\
\hline $97 / 0162$ & $35.17^{\mathrm{bc}}$ & $4.87^{\mathrm{ac}}$ & $33.33^{\mathrm{a}}$ & $6.55^{\text {ad }}$ & $2.04^{\mathrm{bc}}$ & $0.027^{\mathrm{eg} g}$ & $0.26^{\mathrm{ce}}$ \\
\hline $94 / 0034$ & $35.50^{\mathrm{bc}}$ & $4.40^{\mathrm{ac}}$ & $28.33^{\mathrm{a}}$ & $9.14^{\mathrm{ab}}$ & $2.58^{\mathrm{bc}}$ & $0.042^{\mathrm{bc}}$ & $0.53^{\mathrm{abc}}$ \\
\hline $96 / 0603$ & $35.20^{\mathrm{bc}}$ & $5.07^{\mathrm{ac}}$ & $29.67^{\mathrm{a}}$ & $7.05^{\text {ad }}$ & $2.01^{\mathrm{bc}}$ & $0.023^{\text {gh }}$ & $0.22^{\mathrm{de}}$ \\
\hline $96 / 1642$ & $36.20^{\mathrm{bc}}$ & $3.93^{\mathrm{bc}}$ & $25.00^{\mathrm{a}}$ & $7.51^{\mathrm{ac}}$ & $1.93^{\mathrm{bc}}$ & $0.029^{\mathrm{eg} g}$ & $0.27^{\mathrm{ce}}$ \\
\hline $92 / 0067$ & $37.93^{\mathrm{ab}}$ & $4.60^{\mathrm{acc}}$ & $32.00^{\mathrm{a}}$ & $6.00^{\mathrm{add}}$ & $2.05^{\mathrm{bc}}$ & $0.032^{\mathrm{d}-\mathrm{f}}$ & $0.32^{\mathrm{ce}}$ \\
\hline M98/0028 & $34.00^{\mathrm{bc}}$ & $3.87^{\mathrm{c}}$ & $27.33^{\mathrm{a}}$ & $8.00^{\mathrm{ac}}$ & $1.66^{\mathrm{bc}}$ & $0.034^{\mathrm{de}}$ & $0.28^{\mathrm{ce}}$ \\
\hline $92 / 0325$ & $31.83^{\mathrm{c}}$ & $4.87^{\mathrm{ac}}$ & $29.33^{\mathrm{a}}$ & $5.36^{\mathrm{cd}}$ & $1.39^{\mathrm{bc}}$ & $0.033^{\mathrm{d}-\mathrm{f}}$ & $0.22^{\text {de }}$ \\
\hline Mean & $36.50 \pm 0.37$ & $4.72 \pm 0.10$ & $31.52 \pm 1.28$ & $7.37 \pm 0.24$ & $2.19 \pm 0.12$ & $0.03 \pm 0.001$ & $0.35 \pm 0.02$ \\
\hline
\end{tabular}

Means followed by the same letter within the column are not significantly different $(\mathrm{P} \leq 0.05)$ using DMRT

The estimate of phenotypic and genotypic coefficients of variability, heritability (broad-sense) and genetic advance expressed as percentage of grand mean is presented in the Table 5. Generally, the GCV are lower in magnitude than the PCV. Although the magnitude of the variability differed 
from location to location for each character, higher GCV and PCV were recorded for most of the characters at Ibadan compared to Mokwa. The genotypic coefficients of variability ranged from 8.96 for chlorophyll concentration to 30.52 for tuber length in Ibadan and 4.61 for leaf chlorophyll concentration to 31.51 for tuber yield in Mokwa. Heritability estimates (broad-sense) above $60 \%$ was observed for chlorophyll concentration, tuber diameter and length, weight of tuber, dry matter and tuber yield at Ibadan and only dry matter and tuber yield at Mokwa. Low value of genetic advance was observed for chlorophyll concentration (7.09), tuber diameter (4.91) and tuber length (6.09) at Mokwa.

Table 5

Estimates of phenotypic and genotypic coefficient of variability, heritability and genetic advance of yield and its components

\begin{tabular}{|c|c|c|c|c|c|}
\hline Cultivar & Location & $\begin{array}{l}\text { Phenotypic } \\
\text { cient of var }\end{array}$ & $\begin{array}{l}\text { hotypic coeffi- } \\
\text { t of variability }\end{array}$ & Heritability [\%] & $\begin{array}{c}\text { Genetic advance } \\
{[\%]}\end{array}$ \\
\hline \multirow{2}{*}{$\begin{array}{l}\text { Chlorophyll } \\
\text { Concentration }\end{array}$} & Ibadan & 9.74 & 8.96 & 84.64 & 16.99 \\
\hline & Mokwa & 6.17 & 4.61 & 55.74 & 7.09 \\
\hline \multirow{2}{*}{$\begin{array}{l}\text { Tuber } \\
\text { Diameter }\end{array}$} & Ibadan & 11.93 & 10.64 & 79.56 & 19.54 \\
\hline & Mokwa & 9.80 & 4.83 & 24.31 & 4.91 \\
\hline \multirow{2}{*}{$\begin{array}{l}\text { Tuber } \\
\text { Length }\end{array}$} & Ibadan & 31.48 & 30.52 & 93.99 & 60.95 \\
\hline & Mokwa & 13.18 & 6.24 & 22.43 & 6.09 \\
\hline \multirow{2}{*}{$\begin{array}{l}\text { Number } \\
\text { of Tubers }\end{array}$} & Ibadan & 22.44 & 14.76 & 43.26 & 20.00 \\
\hline & Mokwa & 16.70 & 11.11 & 44.26 & 15.23 \\
\hline \multirow{2}{*}{$\begin{array}{l}\text { Weight of Tu- } \\
\text { bers }\end{array}$} & Ibadan & 24.46 & 19.21 & 61.70 & 31.09 \\
\hline & Mokwa & 29.13 & 16.44 & 31.86 & 19.12 \\
\hline \multirow{2}{*}{$\begin{array}{l}\text { Dry } \\
\text { Matter }\end{array}$} & Ibadan & 15.69 & 14.68 & 87.60 & 28.31 \\
\hline & Mokwa & 24.98 & 24.08 & 92.90 & 47.80 \\
\hline \multirow{2}{*}{$\begin{array}{l}\text { Tuber } \\
\text { yield }\end{array}$} & Ibadan & 26.23 & 21.62 & 67.96 & 36.71 \\
\hline & Mokwa & 40.11 & 31.51 & 61.70 & 50.98 \\
\hline
\end{tabular}

Table 6 shows the qualitative description of the 23 cassava genotypes used in the study across the two environments. The observation revealed variability among the cassava genotypes for growth pattern and colour. The growth pattern ranged from short genotypes $(<2 \mathrm{~m})$ with and without branches to tall genotypes $(\geq 2 \mathrm{~m})$ with and without branches. The number of leaf lobes varied from five to six and seven. Leaf, stem and petiole colour ranges from green to greenish-red, reddish-green and purple. Most of the genotypes with above mean tuber yield at Ibadan were related to 
branching growth pattern while there is no specific association of growth pattern with tuber yield in Mokwa.

Qualitative characters of the 23 genotypes of cassava across two environments

\begin{tabular}{|c|c|c|c|c|c|c|c|}
\hline Variety & $\begin{array}{c}\text { Growth } \\
\text { habit }\end{array}$ & $\begin{array}{l}\text { Leaf } \\
\text { colour }\end{array}$ & $\begin{array}{l}\text { Leaf vein } \\
\text { colour }\end{array}$ & $\begin{array}{l}\text { Stem } \\
\text { colour }\end{array}$ & $\begin{array}{l}\text { Petiole } \\
\text { colour }\end{array}$ & $\begin{array}{l}\text { Number } \\
\text { of lobes }\end{array}$ & $\begin{array}{l}\text { Lobe } \\
\text { shape }\end{array}$ \\
\hline $99 / 3073$ & Tall with branches & Dark green & Green & Silver green & Greenish-red & Seven & Lancolate \\
\hline TME419 & Tall without branches & Purple-green & Green & Light brown & Green & Five & Lancolate \\
\hline $95 / 0289$ & Tall without branches & Purple & Reddish-green & Dark brown & Greenish-red & Six & Elliptic \\
\hline $82 / 00058$ & Tall with branches & Purple & Reddish-green & Light brown & Greenish-red & Five & Lancolate \\
\hline $96 / 1565$ & Tall with branches & Purple-green & Green & Silver green & Green & Five & Elliptic \\
\hline M98/0040 & Short without branches & Purple-green & Green & Silver green & Purple & Six & Lancolate \\
\hline 30572 & Tall with branches & Purple & Reddish-green & Dark brown & Green & Five & Lancolate \\
\hline $98 / 0581$ & Short without branches & Purple-green & Green & Silver green & Purple & Six & Elliptic \\
\hline $96 / 1632$ & Short without branches & Purple-green & Green & Light brown & Green & Five & Lancolate \\
\hline $97 / 4769$ & Short with branches & Purple & Reddish-green & Dark brown & Purple & Five & Lancolate \\
\hline $98 / 0510$ & Short with branches & Purple-green & Green & Silver green & Green & Six & Lancolate \\
\hline $4(2) 1425$ & Short with branches & Purple-green & Green & Light brown & Green & Six & Lancolate \\
\hline $92 \mathrm{~B} / 00068$ & Short with branches & Purple & Reddish-green & Dark brown & Purple & Six & Lancolate \\
\hline $91 / 02324$ & Tall with branches & Purple-green & Green & Dark brown & Greenish-red & Five & Elliptic \\
\hline 96/1089A & Short with branches & Purple & Reddish-green & Silver green & Green & Five & Elliptic \\
\hline $98 / 0505$ & Short without branches & Purple-green & Green & Light brown & Greenish-red & Five & Lancolate \\
\hline $97 / 0162$ & Tall without branches & Dark green & Green & Silver green & Greenish-red & Five & Elliptic \\
\hline $94 / 0034$ & Tall without branches & Dark green & Green & Light brown & Green & Six & Lancolate \\
\hline $96 / 0603$ & Tall without branches & Dark green & Green & Light brown & Green & Seven & Lancolate \\
\hline $96 / 1642$ & Tall with branches & Light green & Green & Silver green & Purple & Five & Elliptic \\
\hline $92 / 0067$ & Tall without branches & Purple-green & Green & Dark brown & Purple & Seven & Lancolate \\
\hline M98/0028 & Short without branches & Purple & Reddish-green & Light brown & Purple & Six & Lancolate \\
\hline $92 / 0325$ & Short with branches & Purple & Reddish-green & Dark brown & Purple & Seven & Lancolate \\
\hline
\end{tabular}

Short $(<2 \mathrm{~m})$, Tall $(\geq 2 \mathrm{~m})$

The dendrogram generated from single linkage cluster analysis is presented in Fig. 1. The resultant dendrogram grouped the twenty-three cassava genotypes into four and six main clusters at Ibadan and Mokwa, respectively. The cluster order in the dendrograms was not absolutely identical at the two locations. Genotypes M98/0040 and 92B/0006 were distinct from the other cassava genotypes at Ibadan while 92B/0006, 96/1642, 
92/0325 and 98/0505 were unique at Mokwa. Also, genotypes 96/0289, 96/1565, 96/1632, 91/02324, 98/0510, 98/0581 and 4(2)1425 were grouped together in cluster II and cluster I at Ibadan and Mokwa, respectively.
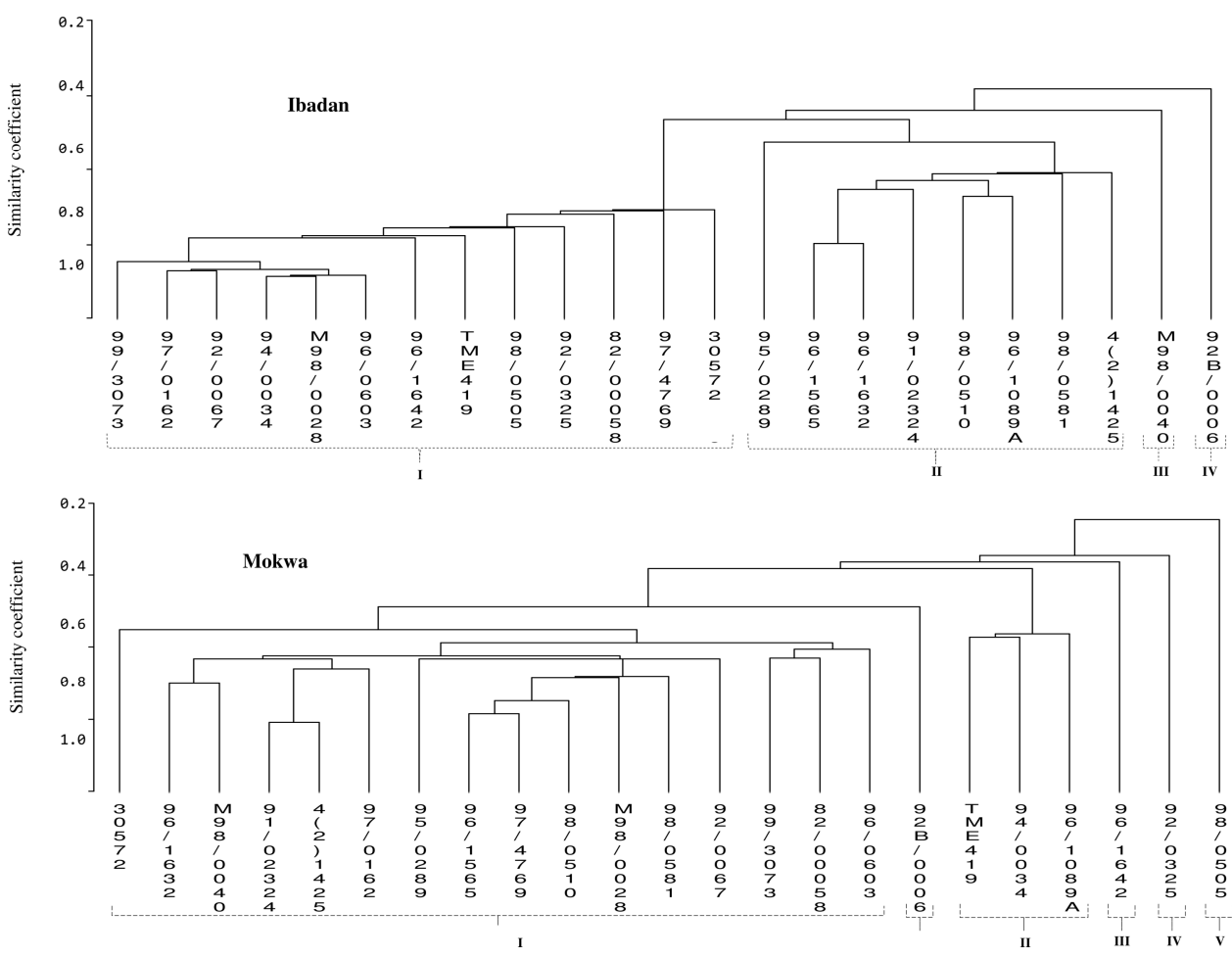

Fig. 1. Cluster analysis dendrogram of the 23 cassava genotypes at the two locations

The means of six cluster groups generated by the FASTCLUS analysis is presented in Table 7 . The pattern of association among the cassava genotypes revealed unique information about their morphological characteristic, and varies across the locations. The range of means revealed a higher performance for most of the characters at Ibadan than Mokwa, however, the means revealed that chlorophyll concentration, tuber length and number of tubers contributed the largest proportion of morphological variation at both locations. At Ibadan, mean value of fresh weight of tubers and tuber yield ranged from group I (2.16 and $0.52 \mathrm{~kg}$, respectively) with nine cassava genotypes to group III (3.70 and $0.81 \mathrm{~kg}$, respectively) with genotype 95/0289 while at Mokwa, it ranged from group IV (0.97 and $0.15 \mathrm{~kg}$, respectively) with two genotypes to group II ( 2.48 and $0.39 \mathrm{~kg}$, respectively) with ten genotypes. The character pattern also reveals that 95/0289 had the highest value for most of the characters evaluated in the study at Ibadan while group II which included 95/0289 and 98/0581 had the highest value for tuber girth, fresh weight of tubers and tuber yield at Mokwa. Also, the 
morphological arrangement at Mokwa reveals a relationship between chlorophyll concentration and dry matter yield. Group III with the highest value of chlorophyll concentration was also characterized with high dry matter while group IV had the lowest value of chlorophyll concentration and dry matter.

Characteristics pattern of six groups of cassava genotypes at the two locations

\begin{tabular}{|c|c|c|c|c|c|c|c|}
\hline \multirow[b]{3}{*}{ Parameters } & Group I & Group II & Group III & Group IV & Group V & Group VI & $\begin{array}{l}\text { Range of } \\
\text { means }\end{array}$ \\
\hline & \multicolumn{7}{|c|}{ Ibadan } \\
\hline & $\begin{array}{c}\text { 82/00058, 92/0067, } \\
\text { 92/0325,94/0034, } \\
\text { 96/0603,96/1642, } \\
\text { 97/0162,99/3073, } \\
\text { TME419 }\end{array}$ & $\begin{array}{c}4(2) 1425 \\
98 / 0581\end{array}$ & 95/0289 & $\begin{array}{c}30572, \\
97 / 4769, \\
98 / 0505\end{array}$ & $\begin{array}{c}\text { 91/02324, } \\
96 / 1089 \mathrm{~A} \\
96 / 1565 \\
96 / 1632 \\
98 / 0510\end{array}$ & $\begin{array}{l}\text { 92B/0006, } \\
\text { M98/0040 }\end{array}$ & \\
\hline $\begin{array}{l}\text { Chlorophyll concen- } \\
\text { tration }\end{array}$ & $33.23(1.40)$ & $32.54(1.19)$ & $38.63(-)$ & $27.72(1.26)$ & $35.86(2.26)$ & $36.97(3.44)$ & $10.91(3.89)$ \\
\hline Tuber girth & $6.59(0.89)$ & $7.33(0.46)$ & $8.20(-)$ & $6.35(0.13)$ & $6.92(0.85)$ & $6.68(0.46)$ & $1.85(0.67)$ \\
\hline Tuber length & $30.10(2.18)$ & $55.17(3.54)$ & $56.00(-)$ & $28.56(3.01)$ & $44.33(3.41)$ & $18.83(4.93)$ & $\begin{array}{c}37.17 \\
(15.32)\end{array}$ \\
\hline Number of tubers & $4.70(1.16)$ & $4.50(0.71)$ & $7.00(-)$ & $4.67(1.15)$ & $4.60(0.89)$ & $4.50(0.71)$ & $2.50(0.99)$ \\
\hline Fresh weight of tubers & $2.16(0.64)$ & $2.56(0.42)$ & $3.70(-)$ & $2.47(0.65)$ & $2.64(0.37)$ & $2.55(0.09)$ & $1.54(0.53)$ \\
\hline Dry matter & $0.048(0.01)$ & $0.045(0.01)$ & $0.040(-)$ & $0.050(0.01)$ & $0.046(0.01)$ & $0.050(-)$ & $0.008(0.21)$ \\
\hline \multirow[t]{2}{*}{ Yield } & $0.52(0.20)$ & $0.62(0.03)$ & $0.81(-)$ & $0.60(0.06)$ & $0.61(0.05)$ & $0.63(0.04)$ & $0.29(0.10)$ \\
\hline & \multicolumn{7}{|c|}{ Mokwa } \\
\hline Parameters & TME419 & $\begin{array}{c}30572,4(2) \\
1425 \\
91 / 02324 \\
92 / 0067 \\
95 / 0289 \\
96 / 1565 \\
96 / 1632 \\
97 / 4769 \\
98 / 0510 \\
98 / 0581\end{array}$ & $\begin{array}{r}\text { 94/0034, } \\
\text { 96/1089A }\end{array}$ & $\begin{array}{c}\text { 92/0325 } \\
\text { 92B/0006 }\end{array}$ & 98/0505 & $\begin{array}{c}\text { 82/00058, } \\
96 / 0603 \\
96 / 1642 \\
99 / 3073 \\
\text { M98/0040, }\end{array}$ & \\
\hline $\begin{array}{l}\text { Chlorophyll concen- } \\
\text { tration }\end{array}$ & $34.41(1.88)$ & $37.08(1.34)$ & $41.70(-)$ & $38.57(-)$ & $37.10(1.39)$ & $34.05(1.92)$ & $7.65(2.82)$ \\
\hline Tuber girth & $4.04(0.24)$ & $4.87(0.41)$ & $4.77(-)$ & $5.30(-)$ & $4.73(0.38)$ & $4.82(0.27)$ & $1.26(0.41)$ \\
\hline Tuber length & $26.67(1.46)$ & $31.61(1.85)$ & $35.67(-)$ & $26.33(-)$ & $39.44(1.39)$ & $30.11(1.07)$ & $13.11(5.14)$ \\
\hline Number of tubers & $8.25(0.50)$ & $7.64(1.03)$ & $7.00(-)$ & $4.00(-)$ & $7.33(0.58)$ & $6.00(1.00)$ & $4.25(1.52)$ \\
\hline Fresh weight of tubers & $2.03(0.39)$ & $2.48(0.73)$ & $2.10(-)$ & $0.97(-)$ & $2.17(0.09)$ & $1.82(0.37)$ & $1.51(0.52)$ \\
\hline Dry matter & $0.030(0.01)$ & $0.032(0.01)$ & $0.040(-)$ & $0.030(-)$ & $0.037(0.01)$ & $0.027(0.01)$ & $0.005(0.01)$ \\
\hline Yield & $0.31(0.16)$ & $0.39(0.16)$ & $0.38(-)$ & $0.15(-)$ & $0.38(0.06)$ & $0.27(0.08)$ & $0.07(0.09)$ \\
\hline
\end{tabular}

Standard deviation in parentheses 


\section{DISCUSSION}

The significant mean square values obtained from the combined analysis of variance for the environmental effect revealed that the conditions in the two locations used in the study were not similar and thus the possibility of having different effect on the performance of the cassava genotypes. The significant genotype $\mathrm{x}$ environment interaction (GEI) observed in most characters also indicated the influence of the environmental condition on the performance of the genotypes. Similar results was observed by Aina et al. (2009) and Akinwale et al. (2011) when they evaluated eighteen and forty-three cassava genotypes, respectively, at different locations in Nigeria.

Comparative performances of the 23 cassava genotypes at the two locations revealed the response of the genotypes to the different agro-climatic conditions. The superiority of some genotypes for a character in one location and not in the other location is a clear indication of the environmental effect. However, breeding potential exists for genotypes 99/3073, TME419, 95/0289, M98/0040, 96/1565, 98/0581, 97/4769, 91/02324, 96/1089A, 94/0034, 96/1632 and 4(2)1425 with above average for yield and most of its related characters in either or both locations. Akinwale et al. (2011) reported the highest value of dry matter yield $(36.45 \%$ - as percentage of fresh weight) and fresh root yield (39.23 kg) for TME419 and 91/02324, respectively, across three agro-climatic zones in Nigeria.

Plant characters show the extent with which selection of genotypes could be based on the phenotype. This study revealed the effect of the environments on genetic diversity and heritability of tuber yield and related traits. Higher GCV and PCV observed in Ibadan for most of the characters showed that the agro-climatic condition in Ibadan reveals more diversity among the cassava genotypes for these characters. This also suggested the reason why higher heritability estimates and genetic advance were recorded for most of the traits at Ibadan. Therefore, the agro-climatic condition in Ibadan allows efficiency of selection through the expression of good cassava genotypes. Varma and Mathra (1993) observed that a high selection gain is expected from dry tuber yield and fresh weight of tubers per plant

In conclusion, the current study revealed genetic variability and differential yield performance among the cassava genotypes across the environments due to the presence of GEI. The interrelationships among the yield and related traits also allowed for the identification of optimal genotypes within each environment. Genotypes 95/0289, 99/3073 and TME419 with high tuber yield in Ibadan and 98/0581, 94/0034 and 97/4769 in Mokwa can be specifically adapted to these agro-climatic zones.

The character pattern among the cassava genotypes can be exploited to improve and develop new cassava genotypes. Also, to optimize the avail- 
able resources, the climatic condition at Ibadan, which favours high genotypic selectivity, should be considered for cassava breeding programmes while cassava genotypes with relatively stable yield across the environments, for instance 95/0289 and 98/0581, can be used for cassava production in the agro-climatic zone.

\section{ACKNOWLEDGEMENTS}

Financial support for this research work was provided by the International Institute of Tropical Agriculture (IITA). The authors also wish to thank Mr. Adeniyi Adetoro (Cassava Unit, IITA) for communicating his valuable experience of the crop.

\section{REFERENCES}

Aina, O. O., Dixon, A. G. O., Paul, I. and Akinrinde, E. A. 2009. G x E interaction effects on yield and yield components of cassava (landraces and improved) genotypes in the savanna regions of Nigeria. Afr. $J$. Biotechnol., 8(19), 4933-4945.

Akinwale, M. G., Akinyele, B. O., Odiyi, A. C. and Dixon A. G. O. 2011. Genotype x environment interaction and yield performance of 43 improved cassava (Manihot esculenta Crantz) genotypes at three agroclimatic zones in Nigeria. British Biotechnology Journal 1(3): 68-84.

Allard, R. W. 1960. Principles of Plant Breeding. John Wiley and Sons, Inc., New York, 485p

FAO (Food and Agricultural Organization) 2002. FAO Trade Statistics. Rome, Italy: FAO.

FAO (Food and Agricultural Organization) 2006. Production data 2006 http://www.fao.org. Accessed October 5,2007

Kawano. K. Fukuda W. M. G. and Cenpukdee, U. 1987. Genetic and environmental effects on dry matter content of cassava tubers. Crop Science 27: 69-74.

Kumar, A., Misra, S. C., Singh, Y. P. and Chauhan, B. P. S 1985. Variability and correlation studies in tuticale, J. Maharashtra Agric Univ. 10: 273-275.

Nweke, F., Spencer. D. S. C. and Lynam, J. K. 2002. The Cassava Transformation, Africa's best kept secret. Michigan State University press, East Lansing, Michigan, USA. p. 273. Shaffi B, Mahler KA, Price WJ, Auld DL.

Olsen, K. M. and Schaal, B. A. 2001. DNA Sequence Data and Inferences on Cassava's Origin of Domestication. In Genetics and Plant Domestication. The Hague Mouton Publishers, Piperno, DR 1995. pp. 123133.

Tsegai, D. and P.C. Kormawa (2002). Determinants of Urban Household Demand for Cassava Products in Kaduna, Northern Nigeria. In: Conference of International Research for Development, Witzenhause, 910 October 2002.

Varma, S. P. and Mathura, R. 1993. Genetic variability and inter-relation in cassava (Manihot escuelnta Crantz) under rained conditions of Tripura. Journal of Tuber Crops. : 19: 77-80. 